\title{
The pencil eraser swab technique to quantify Cutibacterium acnes on shoulder skin
}

\author{
Vendela M. Scheer ${ }^{1}$, Malin Bergman Jungeström² ${ }^{2}$ Lena Serrander ${ }^{3}$, Johan H. Scheer ${ }^{4}$, and \\ Anders Kalén ${ }^{4}$ \\ ${ }^{1}$ Department of Biomedical and Clinical Sciences, Linköping University, Linköping, 581 85, Sweden \\ ${ }^{2}$ Faculty of Health Sciences, Linköping University Hospital, Linköping, 581 85, Sweden \\ ${ }^{3}$ Division of Clinical Microbiology, Department of Biomedical and Clinical Sciences, \\ Linköping University, Linköping, 581 85, Sweden \\ ${ }^{4}$ Division of Orthopedics, Department of Biomedical and Clinical Sciences, Linköping, 581 85, Sweden \\ Correspondence: Vendela M. Scheer (vendela.scheer@liu.se)
}

\begin{abstract}
Received: 23 October 2021 - Revised: 22 November 2021 - Accepted: 26 November 2021 - Published: 17 December 2021
Abstract. Introduction: Cutibacterium acnes is the most common cause of postoperative infections in orthopaedic shoulder surgery and is hard to eradicate with current measures. Newer strategies focus on reducing bacterial load on the skin before surgery. Several previous studies have used a large number of both described and undescribed sampling techniques. The purpose of this study was to compare three previously described swab techniques to obtain bacterial cultures: Levine's (L) technique, the Z technique and the pencil eraser swab (PES) technique. Methods: Three consecutive skin swabs were collected from the right shoulder, on 15 healthy male volunteers, using Levine's technique, $\mathrm{Z}$ technique and PES technique from each participant. To determine the number of living bacteria, serial dilutions were made, and after culturing for $5 \mathrm{~d}$, viable count (VC) was expressed as CFU/mL (with CFU representing colony-forming unit). Results: The PES technique yielded significantly higher VC than the two others. PES: median $3700 \mathrm{CFU} / \mathrm{mL}, \mathrm{L}: 200 \mathrm{CFU} / \mathrm{mL}$ and Z: $220 \mathrm{CFU} / \mathrm{mL}$ $(p=0.003)$. There was no significant difference between the methods regarding the number of positive cultures. PES: $14 / 15$, L: $11 / 15$ and Z: $12 / 15$. Conclusions: There is a need to harmonise sampling techniques of $C$. acnes in order to compare the efficacy of different measures to reduce the bacterial load on the skin before and during surgery. Of the three tested methods, the PES technique is simple and produces the highest bacterial counts.
\end{abstract}

\section{Introduction}

In orthopaedic surgery, surgical site infections (SSIs) are usually caused by the patient's own skin flora, so-called endogenous infection (Krizek and Robson, 1975). Cutibacterium acnes (C. acnes) resides in the sebaceous glands of the skin and are the most common bacteria causing SSI after orthopaedic shoulder surgery (Achermann et al., 2014; Levy et al., 2008; Nelson et al., 2016; Richards et al., 2014). Earlier studies have demonstrated that this species can prevail on the skin despite strict preoperative preparation with alcohol-based chlorhexidine (Lee et al., 2014; Scheer et al., 2021). This has spawned investigation of other eradication strategies, one being to evaluate whether different bactericidal creams applied before surgery can reduce the number of bacteria on the skin (Chalmers et al., 2019; Dizay et al., 2017; Hancock et al., 2018; Murray et al., 2011; Sabetta et al., 2015; Stull et al., 2020) with the presumption that this in turn will reduce bacterial load in the surgical field. Since SSIs in open orthopaedic surgery are relatively rare events $(0.3 \%-$ $5.0 \%$ ) (Singh et al., 2012; Padegimas et al., 2015; Atesok et al., 2017; Eck et al., 2018), showing actual reduction of infection frequency requires tens of thousands of patients in huge multicentre trials. Therefore, commonly, bacterial skin count is indirectly used as an assessment of the effectiveness of a method (Falk-Brynhildsen et al., 2013b; Chalmers et al., 2019; Meyer et al., 2021).

Studies on the subject use different bacterial sampling methods. Several papers use their own, previously non- 
described method (Falk-Brynhildsen et al., 2013a; MacNiven et al., 2018; Blonna et al., 2018), and unfortunately a large number of studies on the subject do not even described what sampling technique is used (Egli-Gany et al., 2012; Murray et al., 2011; Dizay et al., 2017; Matsen et al., 2013; Chuang et al., 2015; Sethi et al., 2015), which makes comparison of results difficult. This is illustrated by the fact that dermal colonisation of $C$. acnes on normal untreated skin in different studies varies between 30\%-97\% (Dizay et al., 2017; Kolakowski et al., 2018; Sabetta et al., 2015; Scheer et al., 2021).

The three most described and used methods for bacterial skin sampling are Levine's technique (Levine et al., 1976), the $\mathrm{Z}$ technique (Angel E Donna et al., 2011) and the swabcup technique (Williamson and Kligman, 1965). The first two were developed to obtain cultures for diagnosing wound infections in clinical practise. The third is not a swab technique in the same sense since a swab is used to stir up a solution in a cylinder held against the skin, and the solution is then aspirated for culture.

We have in two previous studies (Scheer et al., 2018, 2021) introduced yet another method, the PES technique (pencil eraser swab) after executing a number of pilot studies (using chambers, scrapes, changing the number of swab passages, etc.) with the aim to maximise the number of positive cultures with $C$. acnes. The purpose of this study was to compare the PES method to Levine's technique and the $\mathrm{Z}$ technique. This was done with the hypothesis being that the PES technique would yield more positive $C$. acnes cultures and a higher bacterial count as compared to the other two techniques. The swab-cup technique was not investigated since it is too laborious to use in a surgical setting and has produced low viable bacterial counts of anaerobic bacteria (Dorfel et al., 2021).

\section{Materials and methods}

Fifteen healthy male volunteers consented to undergo culture of three skin swabs on unprepared skin. Previous studies have shown that men have more $C$. acnes than women; hence, we included men only (Patel et al., 2009; Dizay et al., 2017; Scheer et al., 2021). Healthy hospital staff members were asked to participate in the study. Written informed consent was obtained from all participants. Inclusion criteria were male, age $>18$ and with legal capacity. Exclusion criteria were any visible skin lesion in the shoulder area or any antimicrobial treatment within $7 \mathrm{~d}$ of the swabbing (to maximise bacterial count).

Three consecutive skin swabs were collected from the right shoulder, using the $\mathrm{L}$ technique, $\mathrm{Z}$ technique and PES technique from each participant. Three different sterile templates, nos. 1, 2 or 3, were used to ensure equal swabbing areas in all subjects (Fig. 1). The template number used for each volunteer was chosen by randomly selecting it from an
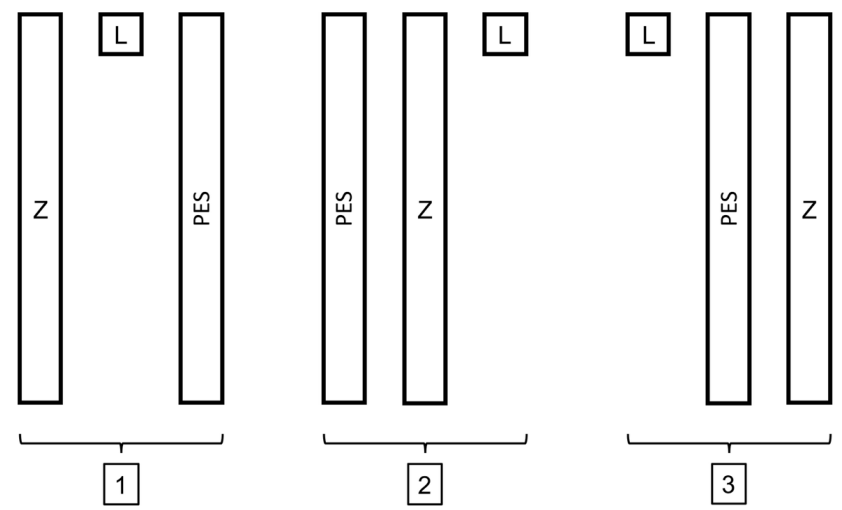

Figure 1. The three different templates. Z: Z technique, L: Levine's technique and PES: pencil eraser swab technique. Each rectangle has the dimensions of $1 \mathrm{~cm} \times 10 \mathrm{~cm}$, the square measures $1 \mathrm{~cm} \times$ $1 \mathrm{~cm}$, and there is $1 \mathrm{~cm}$ between each template.

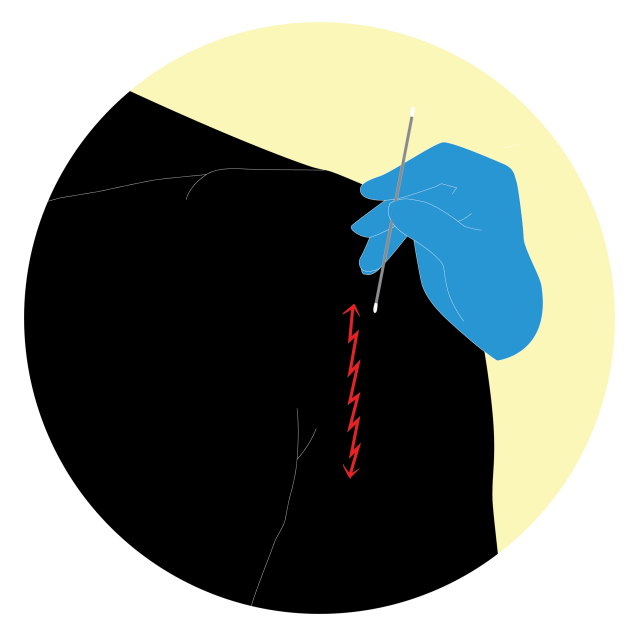

Figure 2. PES technique: rub the swab with an oscillating movement - like using a pencil eraser - going down over a $10 \mathrm{~cm}$ line and then in the same manner up again for a total of 15 passages (https://doi.org/10.5446/55554; Scheer, 2021a).

envelope. For swabbing, we use eSwab (Copan Italia S.p.A. via Perotti 10, Brescia, Italy), a flocked swab with a tube, containing $1 \mathrm{~mL}$ of liquid Amies, which elutes the entire sample into the medium.

\subsection{Sample techniques}

The $\mathrm{Z}$ technique involves rotating the swab in a 10-point zigzag fashion once - in this study over a $10 \mathrm{~cm}$ line, corresponding to a standard shoulder incision. Levine's technique ( $\mathrm{L}$ technique) consists of rotating the swab over a $1 \mathrm{~cm}$ area with "sufficient" pressure for $5 \mathrm{~s}$. Finally, the PES technique: rub the swab with an oscillating movement - like using a pencil eraser - going down over a $10 \mathrm{~cm}$ line and then in the same manner up again for a total of 15 passages (Fig. 2). 
Table 1. Qualitative results; subjects with positive bacterial cultures $(n=15)$.

\begin{tabular}{llll}
\hline Bacteria & PES technique & Levine's technique & Z technique \\
\hline C. acnes (no. of positive cultures) & $14 / 15$ & $11 / 15$ & $12 / 15$ \\
CoNS (no. of positive cultures) & $11 / 15$ & $9 / 15$ & $8 / 15$ \\
Median viable count [CFU/mL] (Range) & $3700(140-133000)$ & $200(0-6600)$ & $220(0-4300)$ \\
\hline
\end{tabular}

C.: Cutibacterium, CoNS: coagulase-negative staphylococcus

Table 2. Quantitative results in pairwise comparisons.

\begin{tabular}{lrr}
\hline $\begin{array}{l}\text { Pairwise comparison } \\
\text { between groups }\end{array}$ & $\begin{array}{r}\text { Significance } \\
\text { level }\end{array}$ & $\begin{array}{r}\text { Adjusted } \\
\text { significance* }\end{array}$ \\
\hline L and Z techniques & 0.813 & 1.00 \\
L and PES techniques & 0.004 & 0.013 \\
Z and PES techniques & 0.002 & 0.006 \\
\hline
\end{tabular}

* Adjustment by the Bonferroni correction for multiple tests

\subsection{Microbiological technique}

All skin swabs were immediately put into the medium and within $1 \mathrm{~h}$, transported to the laboratory; they were vortexed for $10 \mathrm{~s}$ whereafter serial dilutions were made, and these were cultured on anaerobic agar medium without antibiotics and placed in an anaerobic incubator. After $5 \mathrm{~d}$, we counted the colony-forming units (CFU), and viable count (VC) is expressed as CFU/mL (Ben-David and Davidson, 2014). Bacteria species were detected with matrix-assisted laser desorption/ionisation time-of-flight mass spectrometry (MALDITOF).

\subsection{Statistical analyses}

Patzer, Phadnis and Falk-Brynhildsen (Patzer et al., 2018; Phadnis et al., 2016; Falk-Brynhildsen et al., 2013a) all used different swab techniques that yielded on average $42 \%$ positive cultures of $C$. acnes. In two previous studies, using the PES technique, we had $97 \%$ positive cultures of C. acnes (Scheer et al., 2018, 2021). With an $80 \%$ power and a significance level of 0.05 , a sample size of 15 subjects was required. We used the chi-square test for categorial variables. The Kruskal-Wallis test for ranks (one-way ANOVA on ranks) was used for comparing distributions with a following pairwise comparison with adjustment by the Bonferroni correction for multiple tests.

\section{Results}

Fifteen male volunteers were enrolled in this study. Their average age was 46 (range 28-65). There were no significant differences between the different techniques in detecting $C$. acnes (positive cultures) or CoNS (coagulase-negative staphylococci) (Table 1). The CoNS found were identified as
Staphylococcus epidermidis, S. saccarolyticus, S. hominis, $S$. cristatus and $S$. capitis. The one-way ANOVA on ranks showed that the distribution of $\mathrm{VC}$ was not the same across categories of groups $(p=0.003)$. The pairwise comparisons are displayed in Table 2 demonstrating the PES technique producing significantly higher viable counts with no difference between the $\mathrm{Z}$ and $\mathrm{L}$ techniques. In all techniques, $84 \%-87 \%$ of the CFUs were $C$. acnes.

\section{Discussion}

Our results suggest that the PES technique is effective in detecting high quantities of viable $C$. acnes compared to Levine's and the $\mathrm{Z}$ techniques. Hence, it may be more usable when evaluating measures to reduce bacterial load on the skin prior to surgery - at least for C. acnes.

When studying different aseptic preoperative preparations, it is important to have sensitive and reproducible methods. Results from earlier studies display considerable variation identifying $C$. acnes on the skin before preparation (Table 3). The true rate of $C$. acnes colonisation in the area is unknown, but we believe it is close to $100 \%$ based on previous work on the microbiome of the skin (Huse et al., 2012). It is paramount that studies designed to evaluate preoperative preparation and its efficacy on reducing bacterial load describe the method transparently and completely. Even so, it is difficult to detect differences in preoperative techniques if the method in question has low sensitivity. Tape stripping, surface scrapes and cup-scrub technique are techniques that have been documented and validated for skin microbiome sampling (Kong et al., 2017; Chng et al., 2016). In clinical settings, the cup-scrub technique can be cumbersome to use, and the tape-stripping method may cause skin damage, making it unfit in a surgical setting. An optimal method must leave the skin uninjured by the sampling; otherwise, it could increase the risk of an SSI if the samples are taken perioperatively. The eSwab is inexpensive and the simplicity of the method makes it easy to use. Parada et al. (2018) point to the lack of consensus in prevention of shoulder arthroplasty infection in a survey and also that we need to create best practice guidelines to limit SSI after shoulder surgery. 
Table 3. Positive $C$. acnes cultures in different studies.

\begin{tabular}{lrrrrl}
\hline Primary study & $\begin{array}{r}\text { No. of } \\
\text { patients }\end{array}$ & $\begin{array}{r}\text { Male } \\
(\%)\end{array}$ & $\begin{array}{r}\text { Female } \\
(\%)\end{array}$ & $\begin{array}{r}\text { Positive } \text {. acnes cultures } \\
\text { before treatment }\end{array}$ & $\begin{array}{l}\text { Swab } \\
\text { technique }\end{array}$ \\
\hline Chuang et al. (2015) & 51 & 74 & 26 & $72 \%$ & ND \\
Phadnis et al. (2016) & 50 & 60 & 40 & $42 \%$ & ND \\
Murray et al. (2011) & 50 & 50 & 50 & $58 \%$ & ND \\
Dizay et al. (2017) & 65 & 66 & 34 & $48 \%$ & ND \\
Matsen et al. (2013) & 30 & 60 & 40 & $77 \%$ & ND \\
Sabetta et al. (2015) & 50 & 46 & 54 & $32 \%$ & ND \\
Scheer et al. (2018) & 40 & 60 & 40 & $95 \%$ & PES \\
Scheer et al. (2021) & 100 & 63 & 37 & $97 \%$ & PES \\
\hline
\end{tabular}

ND: not described, PES: pencil eraser swab technique

\section{Limitations}

The range of VC is large in all groups. Hopefully this reflects a difference in true bacterial load. The sampling was performed at different times during the day, but it was not noted in the protocol how long before the sampling that the subjects had showered. However, this would affect the VC in all techniques equally since all the sampling in each subject was performed at the same time. Also, comparison of VC was made statistically pairwise resulting in each subject acting as their own control unless there is a substantial local variation of the bacterial load within the $5 \mathrm{~cm} \times 10 \mathrm{~cm}$ dimensions of the template. This seems unlikely but cannot be completely ruled out. It must be noted that we have sampled $C$. acnes on the skin when we really want to assess dermal bacterial load. We only presume that they correlate, but this has not, to our knowledge, been shown. It appears, however, highly likely that since $C$. acnes reside and thrive in the sebaceous glands and not superficially, a high skin count reflects a high dermal count.

We have used the PES method in a clinical study without obvious skin abrasion (Scheer et al., 2021), but we believe that rubbing with swabs with any method should be used with caution or not at all on delicate skin in conjunction with surgery in the area.

\section{Conclusion}

To our knowledge, this is the first study comparing different skin swab techniques on healthy skin. The PES technique is easy to use, appears effective in detecting $C$. acnes and gives a high bacterial yield. It could be used in future studies to evaluate preoperative measures.

Ethical statement. Ethical review agency approval has been received from the Swedish Ethical Review Authority (2016-488-31, 2019-05613).
Code availability. Data analyses were performed by using IBM $^{\circledR}$ SPSS $^{\circledR}$ Statistics.

Data availability. Underlying research data can be accessed by https://doi.org/10.5281/zenodo.5774118 (Scheer, 2021b).

Video supplement. The video supplement related to this article is available online at https://doi.org/10.5446/55554 (Scheer, 2021a).

Supplement. The supplement related to this article is available online at: https://doi.org/10.5194/jbji-6-451-2021-supplement.

Author contributions. All authors contributed to the study's conception and design. Data collection was performed by VMS. Analyses were performed by VMS and JHS. The first draft of the manuscript was written by VMS. All authors read and approved the final article.

Competing interests. The contact author has declared that neither they nor their co-authors have any competing interests.

Disclaimer. Publisher's note: Copernicus Publications remains neutral with regard to jurisdictional claims in published maps and institutional affiliations.

Review statement. This paper was edited by Parham Sendi and reviewed by two anonymous referees.

\section{References}

Achermann, Y., Goldstein, E. J., Coenye, T., and Shirtliff, M. E.: Propionibacterium acnes: from commensal to opportunistic biofilm-associated implant pathogen, Clin. Microbiol. Rev., 27, 419-440, https://doi.org/10.1128/CMR.00092-13, 2014. 
Angel E Donna, L. P., Carville, K., and Santamaria, N.: The clinical efficacy of two semi-quantitative wound-swabbing techniques in identifying the causative organism(s) in infected cutaneous wounds, Int. Wound J., 8, 176-185, 2011.

Atesok, K., MacDonald, P., Leiter, J., McRae, S., Stranges, G., and Old, J.: Postoperative deep shoulder infections following rotator cuff repair, World J. Orthop., 8, 612-618, https://doi.org/10.5312/wjo.v8.i8.612, 2017.

Ben-David, A. and Davidson, C. E.: Estimation method for serial dilution experiments, J. Microbiol. Methods, 107, 214-221, https://doi.org/10.1016/j.mimet.2014.08.023, 2014.

Blonna, D., Allizond, V., Bellato, E., Banche, G., Cuffini, A. M., Castoldi, F., and Rossi, R.: Single versus Double Skin Preparation for Infection Prevention in Proximal Humeral Fracture Surgery, Biomed. Res. Int., 2018, 8509527, https://doi.org/10.1155/2018/8509527, 2018.

Chalmers, P. N., Beck, L., Stertz, I., and Tashjian, R. Z.: Hydrogen peroxide skin preparation reduces Cutibacterium acnes in shoulder arthroplasty: a prospective, blinded, controlled trial, J. Shoulder Elbow Surg., 28, 1554-1561, https://doi.org/10.1016/j.jse.2019.03.038, 2019.

Chng, K. R., Tay, A. S., Li, C., Ng, A. H., Wang, J., Suri, B. K., Matta, S. A., McGovern, N., Janela, B., Wong, X. F., Sio, Y. Y., Au, B. V., Wilm, A., De Sessions, P. F., Lim, T. C., Tang, M. B., Ginhoux, F., Connolly, J. E., Lane, E. B., Chew, F. T., Common, J. E., and Nagarajan, N.: Whole metagenome profiling reveals skin microbiome-dependent susceptibility to atopic dermatitis flare, Nat. Microbiol., 1, 16106, https://doi.org/10.1038/nmicrobiol.2016.106, 2016.

Chuang, M. J., Jancosko, J. J., Mendoza, V., and Nottage, W. M.: The Incidence of Propionibacterium acnes in Shoulder Arthroscopy, Arthroscopy, J. Arthrosc. Rel. Surg., 31, 17021707, https://doi.org/10.1016/j.arthro.2015.01.029, 2015.

Dizay, H. H., Lau, D. G., and Nottage, W. M.: Benzoyl peroxide and clindamycin topical skin preparation decreases Propionibacterium acnes colonization in shoulder arthroscopy, J. Shoulder Elbow Surg., S1058-2746(17)30148-9, https://doi.org/10.1016/j.jse.2017.03.003, 2017.

Dorfel, D., Maiwald, M., Daeschlein, G., Muller, G., Hudek, R., Assadian, O., Kampf, G., Kohlmann, T., Harnoss, J. C., and Kramer, A.: Comparison of the antimicrobial efficacy of povidone-iodine-alcohol versus chlorhexidine-alcohol for surgical skin preparation on the aerobic and anaerobic skin flora of the shoulder region, Antimicrob. Resist. Infect. Control., 10, 17, https://doi.org/10.1186/s13756-020-00874-8, 2021.

Eck, C. F., Neumann, J. A., Limpisvasti, O., and Adams, C. R.: Lack of level I evidence on how to prevent infection after elective shoulder surgery, Knee Surg. Sports Traumatol. Arthrosc., 26, 2465-2480, https://doi.org/10.1007/s00167-018-4832-7, 2018.

Egli-Gany, D., Hintzpeter, M., and Andrée, S.: Evaluation of the Antiseptic Efficacy and Local Tolerability of a Polihexanide-Based Antiseptic on Resident Skin Flora, Adv. Skin Wound Care, 25, 404-408, https://doi.org/10.1097/01.ASW.0000419405.52570.3e, 2012.

Falk-Brynhildsen, K., Friberg, O., Soderquist, B., and Nilsson, U. G.: Bacterial colonization of the skin following aseptic preoperative preparation and impact of the use of plastic adhesive drapes, Biol. Res. Nurs., 15, 242-248, https://doi.org/10.1177/1099800411430381, 2013a.
Falk-Brynhildsen, K., Soderquist, B., Friberg, O., and Nilsson, U. G.: Bacterial recolonization of the skin and wound contamination during cardiac surgery: a randomized controlled trial of the use of plastic adhesive drape compared with bare skin, J. Hosp. Infect., 84, 151-158, https://doi.org/10.1016/j.jhin.2013.02.011, 2013b.

Hancock, D. S., Rupasinghe, S. L., Elkinson, I., Bloomfield, M. G., and Larsen, P. D.: Benzoyl peroxide + chlorhexidine versus chlorhexidine alone skin preparation to reduce Propionibacterium acnes: a randomized controlled trial, ANZ J. Surg., 88, 1182-1186, https://doi.org/10.1111/ans.14848, 2018.

Huse, S. M., Ye, Y., Zhou, Y., and Fodor, A. A.: A core human microbiome as viewed through $16 \mathrm{~S}$ rRNA sequence clusters, PLoS One, 7, e34242, https://doi.org/10.1371/journal.pone.0034242, 2012.

Kolakowski, L., Lai, J. K., Duvall, G. T., Jauregui, J. J., Dubina, A. G., Jones, D. L., Williams, K. M., Hasan, S. A., Henn 3rd, R. F., and Gilotra, M. N.: Neer Award 2018: Benzoyl peroxide effectively decreases preoperative Cutibacterium acnes shoulder burden: a prospective randomized controlled trial, J. Shoulder Elbow Surg., 27, 1539-1544, https://doi.org/10.1016/j.jse.2018.06.012, 2018.

Kong, H. H., Andersson, B., Clavel, T., Common, J. E., Jackson, S. A., Olson, N. D., Segre, J. A., and TraidlHoffmann, C.: Performing Skin Microbiome Research: A Method to the Madness, J. Invest. Dermatol., 137, 561-568, https://doi.org/10.1016/j.jid.2016.10.033, 2017.

Krizek, T. J. and Robson, M. C.: Biology of surgical infection, Surg. Clin. North Am., 55, 1261-1267, https://doi.org/10.1016/s00396109(16)40779-6, 1975.

Lee, M. J., Pottinger, P. S., Butler-Wu, S., Bumgarner, R. E., Russ, S. M., and Matsen, F. A.: Propionibacterium Persists in the Skin Despite Standard Surgical Preparation, J. Bone Joint Surg., 96, 1447-1450, https://doi.org/10.2106/jbjs.m.01474, 2014.

Levine, N. S., Lindberg, R. B., Mason Jr., A. D., and Pruitt Jr., B. A.: The quantitative swab culture and smear: A quick, simple method for determining the number of viable aerobic bacteria on open wounds, J. Trauma, 16, 89-94, 1976.

Levy, P. Y., Fenollar, F., Stein, A., Borrione, F., Cohen, E., Lebail, B., and Raoult, D.: Propionibacterium acnesPostoperative Shoulder Arthritis: An Emerging Clinical Entity, Clin. Infect. Dis., 46, 1884-1886, https://doi.org/10.1086/588477, 2008.

MacNiven, I., Hsu, J. E., Neradilek, M. B., and Matsen 3rd, F. A.: Preoperative Skin-Surface Cultures Can Help to Predict the Presence of Propionibacterium in Shoulder Arthroplasty Wounds, JB JS Open Access, 3, e0052, https://doi.org/10.2106/JBJS.OA.17.00052, 2018.

Matsen 3rd, F. A., Butler-Wu, S., Carofino, B. C., Jette, J. L., Bertelsen, A., and Bumgarner, R.: Origin of propionibacterium in surgical wounds and evidence-based approach for culturing propionibacterium from surgical sites, J. Bone Joint Surg., 95, e1811-1817, https://doi.org/10.2106/JBJS.L.01733, 2013.

Meyer, L. E., Lazarides, A. L., Hendren, S., Lassiter, T., Klifto, C., and Anakwenze, O.: The Efficacy of Peroxide Solutions in Decreasing Cutibacterium acnes Burden Around the Shoulder, J. Am. Acad. Orthop. Surg., 2021, 1-8, https://doi.org/10.5435/JAAOS-D-21-00457, 2021.

Murray, M. R., Saltzman, M. D., Gryzlo, S. M., Terry, M. A., Woodward, C. C., and Nuber, G. W.: Efficacy of preoperative home use of $2 \%$ chlorhexidine gluconate cloth be- 
fore shoulder surgery, J. Shoulder Elbow Surg., 20, 928-933, https://doi.org/10.1016/j.jse.2011.02.018, 2011.

Nelson, G. N., Davis, D. E., and Namdari, S.: Outcomes in the treatment of periprosthetic joint infection after shoulder arthroplasty: a systematic review, J. Shoulder Elbow Surg., 25, 1337-1345, https://doi.org/10.1016/j.jse.2015.11.064, 2016.

Padegimas, E. M., Maltenfort, M., Ramsey, M. L., Williams, G. R., Parvizi, J., and Namdari, S.: Periprosthetic shoulder infection in the United States: incidence and economic burden, J. Shoulder Elbow Surg., 24, 741-746, https://doi.org/10.1016/j.jse.2014.11.044, 2015.

Parada, S. A., Shaw, K. A., Eichinger, J. K., Stadecker, M. J., Higgins, L. D., and Warner, J. J. P.: Survey of shoulder arthroplasty surgeons' methods for infection avoidance of Propionibacterium, J. Orthop., 15, 177-180, https://doi.org/10.1016/j.jor.2018.01.052, 2018.

Patel, A., Calfee, R. P., Plante, M., Fischer, S. A., and Green, A.: Propionibacterium acnes colonization of the human shoulder, J. Shoulder Elbow Surg., 18, 897-902, https://doi.org/10.1016/j.jse.2009.01.023, 2009.

Patzer, T., Petersdorf, S., Krauspe, R., Verde, P. E., Henrich, B., and Hufeland, M.: Prevalence of Propionibacterium acnes in the glenohumeral compared with the subacromial space in primary shoulder arthroscopies, J. Shoulder Elbow Surg., 27, 771-776, https://doi.org/10.1016/j.jse.2017.10.039, 2018.

Phadnis, J., Gordon, D., Krishnan, J., and Bain, G. I.: Frequent isolation of Propionibacterium acnes from the shoulder dermis despite skin preparation and prophylactic antibiotics, J. Shoulder Elbow Surg., 25, 304-310, https://doi.org/10.1016/j.jse.2015.08.002, 2016.

Richards, J., Inacio, M. C., Beckett, M., Navarro, R. A., Singh, A., Dillon, M. T., Sodl, J. F., and Yian, E. H.: Patient and procedure-specific risk factors for deep infection after primary shoulder arthroplasty, Clin. Orthop. Relat. Res., 472, 2809-2815, https://doi.org/10.1007/s11999-014-3696-5, 2014.

Sabetta, J. R., Rana, V. P., Vadasdi, K. B., Greene, R. T., Cunningham, J. G., Miller, S. R., and Sethi, P. M.: Efficacy of topical benzoyl peroxide on the reduction of Propionibacterium acnes during shoulder surgery, J. Shoulder Elbow Surg., 24, 995-1004, https://doi.org/10.1016/j.jse.2015.04.003, 2015.
Scheer, V.: The Pencil Eraser Swab Technique, TIB [video supplement], https://doi.org/10.5446/55554, 2021a.

Scheer, V. M.: The Pencil Eraser Swab Technique to Quantify Cutibacterium acnes on Shoulder Skin, Zenodo [data set], https://doi.org/10.5281/zenodo.5774118, 2021 b.

Scheer, V. M., Bergman Jungestrom, M., Lerm, M., Serrander, L., and Kalen, A.: Topical benzoyl peroxide application on the shoulder reduces Propionibacterium acnes: a randomized study, J. Shoulder Elbow Surg., 27, 957-961, https://doi.org/10.1016/j.jse.2018.02.038, 2018.

Scheer, V. M., Jungeström, M. B., Serrander, L., Kalén, A., and Scheer, J. H.: Benzoyl peroxide treatment decreases Cutibacterium acnes in shoulder surgery, from skin incision until wound closure, J. Shoulder Elbow Surg., 30, 1316-1323, https://doi.org/10.1016/j.jse.2020.12.019, 2021.

Sethi, P. M., Sabetta, J. R., Stuek, S. J., Horine, S. V., Vadasdi, K. B., Greene, R. T., Cunningham, J. G., and Miller, S. R.: Presence of Propionibacterium acnes in primary shoulder arthroscopy: results of aspiration and tissue cultures, J. Shoulder Elbow Surg., 24, 796-803, https://doi.org/10.1016/j.jse.2014.09.042, 2015.

Singh, J. A., Sperling, J. W., Schleck, C., Harmsen, W. S., and Cofield, R. H.: Periprosthetic infections after total shoulder arthroplasty: a 33-year perspective, J. Shoulder Elbow Surg., 21, 1534-1541, https://doi.org/10.1016/j.jse.2012.01.006, 2012.

Stull, J. D., Nicholson, T. A., Davis, D. E., and Namdari, S.: Addition of $3 \%$ hydrogen peroxide to standard skin preparation reduces Cutibacterium acnes-positive culture rate in shoulder surgery: a prospective randomized controlled trial, J. Shoulder Elbow Surg., 29, 212-216, https://doi.org/10.1016/j.jse.2019.09.038, 2020.

Williamson, P. and Kligman, A. M.: A New Method for the Quantitative Investigation of Cutaneous Bacteria, J. Invest. Dermatol., 45, 498-503, https://doi.org/10.1038/jid.1965.164, 1965. 